\title{
Coherent atomic oscillations and resonances between coupled Bose-Einstein condensates with time-dependent trapping potential
}

\author{
F. Kh. Abdullaev \\ Physical-Technical Institute, Uzbek Academy of Sciences, G. Mavlyanov Street, 2-b, 700084 Tashkent-84, Uzbekistan \\ and Instituto de Física Teorica, Universidade Estadual Paulista, Rua Pamplona 145, 01405-900 São Paulo, Brazil \\ R. A. Kraenkel \\ Instituto de Física Teorica, Universidade Estadual Paulista, Rua Pamplona 145, 01405-900 São Paulo, Brazil
}

(Received 1 March 2000; published 20 July 2000)

\begin{abstract}
We study the quantum coherent tunneling between two Bose-Einstein condensates separated through an oscillating trap potential. The cases of slow and rapid varying in the time trap potential are considered. In the case of a slowly varying trap, we study the nonlinear resonances and chaos in the oscillations of the relative atomic population. Using the Melnikov function approach, we find the conditions for chaotic macroscopic quantum-tunneling phenomena to exist. Criteria for the onset of chaos are also given. We find the values of frequency and modulation amplitude which lead to chaos on oscillations in the relative population, for any given damping and the nonlinear atomic interaction. In the case of a rapidly varying trap, we use the multiscale expansion method in the parameter $\varepsilon=1 / \Omega$, where $\Omega$ is the frequency of modulations, and we derive the averaged system of equations for the modes. The analysis of this system shows that new macroscopic quantum self-trapping regions, in comparison with the constant trap case, exist.

PACS number(s): 05.30.Jp
\end{abstract}

\section{INTRODUCTION}

It has recently been shown that there exists a macroscopic quantum phase difference in processes connected with atomic waves. Namely, this effect is observed between two tunnel coupled Bose-Einstein condensates [1-3]. The coupling occurs due to a trap potential which has the form of a double-well potential. The theoretical investigation shows that there is possibly an interesting phenomenon of periodic oscillations of the atomic population between condensates and quantum self-trapping of population in the dependence of the relative phase between condensates [4-8]. Analogous phenomena which have been studied are the ac Josephson effect [9] and the periodic exchange of power and switching of electromagnetic waves between cores in nonlinear optical couplers [10].

In particular, there is a direct analogy between the tunneling phenomena in two prolongated Bose-Einstein condensates and two tunnel-coupled single-mode optical fibers. In this optical analogy, the role of the chemical potential is played by the propagation constant, and the nonlinear interaction between atoms is analogous to the Kerr nonlinearity of optical media. The tunnel coupling arising from the overlaps of the electromagnetic fields outside of dielectric cilinders (fibers) [11-13] exactly corresponds to the tunnel coupling between two Bose-Einstein condensates due to the overlaps of the wave functions.

In this context, it is natural to investigate the influence of a time-varying coupling on the quantum coherent tunneling process. In the nonlinear optical coupler analogy, this corresponds to the variation in the longitudinal direction coupling $[14,15]$. As was shown recently, the variation in time of the trap potential can lead to resonant oscillations of the BoseEinstein condensate $[16,17]$. The numerical solution of the Gross-Pitaevsky equation shows that the rapid variation in time of the trap potential can lead to a bifurcation of the effective (averaged) form of the trap potential and consequently to splitting of the BEC [18].

From what we have said above, we could wait for new phenomena in the process of quantum coherent atomic tunneling (QCAT) too. Recalling the analogy with Josephson effects in coupled junctions, we note that in the last case it is very difficult to produce such effects, the difficulty coming from the fact that it is hard to implement the time-varying overlap properties of junctions.

Thus, in this paper we will study the atomic tunneling between two tunnel-coupled BEC's in a double-well timedependent trap. Namely, in Sec. II we will study the nonlinear resonance phenomena in the interference and, in particular, the stationary regimes, with damping taken into account. The phase and population damping are considered as well. The phase-locked states are interesting because of the suppression in these states of the fluctuations of the relative phase between two condensates. The regions of regular and chaotic oscillations of the relative atomic population are calculated in Sec. III, using the Melnikov function approach. In Sec. IV the macroscopic quantum interference in two overlapped condensates with rapidly varying traps is investigated. We derive the system of averaged equations for coupled modes and define in Sec. V new regimes of parameters for the macroscopic quantum self-trapping (MQST).

\section{FORMULATION OF PROBLEM. SLOWLY VARYING IN TIME TRAP}

The problem of Bose-Einstein condensates in a twodouble-well time-dependent trap can be described by the following model: 


$$
\begin{gathered}
i h \frac{\partial \psi_{1}}{\partial t}=\left[E_{1}(t)+\alpha_{1}\left|\psi_{1}\right|^{2}\right] \psi_{1}-K(t) \psi_{2}, \\
i h \frac{\partial \psi_{2}}{\partial t}=\left[E_{2}(t)+\left.\alpha_{2} \psi_{2}\right|^{2}\right] \psi_{2}-K(t) \psi_{1},
\end{gathered}
$$

where the parameters $E_{i}, \alpha_{i}, K(t)$ are defined by the overlap integrals of the time-dependent Gross-Pitaevsky eigenfunctions as given in [4]. $\alpha_{i}$ are parameters of nonlinear interactions between atoms and $\alpha_{i} \sim g_{0}=4 \pi h^{2} a / m$, where $a$ is the atomic scattering length. The attractive case can be obtained analogously, using symmetries of the equation. This system of equations is valid in the approximation of a weak link between condensates. Comparison with the numerical solution of GPE shows that the system (1) is a reasonable approximation for $z \leqslant 0.5-0.6$. For the strongly overlapped condensates, the system should be modified. For a periodically varying $K(t)=K_{0}+K_{1} \sin (\Omega t)$ we have limitations on the parameters $K_{1}$ and $\Omega$ for which the two-mode approximation is valid. The small deformation of bound states requires $K_{1} \ll K_{0}$. To avoid the resonances of the modulations with normal modes of the spherical well, we should require that the difference of energy between the ground state and the first normal mode be larger than the modulation energy, i.e., $\delta E \gg \hbar \Omega$. Taking into account that $\delta E \sim \hbar \omega_{0}[7]$, where $\omega_{0}$ is the harmonic-oscillator frequency, we find the condition $\Omega \ll \omega_{0}$. Taking into account that $\omega_{0} \gg \omega_{L}=2 K_{0}$ - the frequency of the linear oscillations of the atomic population, defined by the tunneling frequency-we conclude that by a proper choice of parameters we can have the resonant case $\Omega \simeq \omega_{L}$ or the rapidly varying modulation $\Omega \gg \omega_{L}$.

Introducing new variables $u_{i}=\sqrt{N_{i}} \exp \left(i \theta_{i}\right), \quad z=\left(N_{1}\right.$ $\left.-N_{2}\right) / N_{T}, N_{T}=N_{1}+N_{2}$, and $\psi=\theta_{1}-\theta_{2}$, where $N_{i}$, and $\theta_{i}$ are, respectively, the number of atoms and phases in the $i$ th trap, we get the following system:

$$
\begin{gathered}
z_{t}=-2 K(t) \sqrt{1-z^{2}} \sin \Phi-\eta \Phi_{t}, \\
\Phi_{t}=\nu \Lambda z+\frac{2 K(t) z}{\sqrt{1-z^{2}}} \cos \Phi+\Delta E(t) .
\end{gathered}
$$

where $\Lambda=\left(\alpha_{1}+\alpha_{2}\right) N_{T}$ and $\nu= \pm 1$ for the positive and negative atomic scattering length, respectively. In what follows, we will consider the case $\nu=1\left(a_{s}>0\right)$, if not stated otherwise. We have included in Eq. (2) the damping term $\eta \phi_{t}(t)$. It appears if we take into account a noncoherent dissipative current of normal-state atoms, proportional to the chemical potential difference $\Delta \mu$. For the other type of overlapping condensate, there might be another type of damping. For example, for the two interacting condensates with different hyperfine levels in a single harmonic trap, the damping has the form $\eta z(t)$.

The Hamiltonian of the unperturbed system $[K(t)$ $=$ const, $\Delta E=$ const, $\eta=0]$ is

$$
H=\frac{\Lambda z^{2}}{2}-2 K \sqrt{1-z^{2}} \cos (\Phi)+\Delta E z
$$

Let us consider the case of periodic modulations of the tunnel coupling parameter, when $K(t)=K_{0}+K_{1} \sin \Omega t$ and the time-periodic energy difference is $\Delta E(t)=\Delta E$ $+\Delta E_{1} \sin (\Omega t)$. It is useful to introduce the parameter $\Delta$ $=\Phi-\Omega t$, which is a slow-varying function of the time in a comparison with the period $\Omega$. The averaged system of equations can be found by averaging over the period of fast oscillations $2 \pi / \Omega$. Doing so, we get the system

$$
\begin{aligned}
& \bar{z}_{t}=-K_{1} \cos \bar{\Delta} \sqrt{1-\bar{z}^{2}}-\eta \Delta_{t}-\eta \Omega, \\
& \bar{\Delta}_{t}=-\Omega+\Delta E+\Lambda z-\frac{K_{1} z}{\sqrt{1-\bar{z}^{2}}} \sin \bar{\Delta} .
\end{aligned}
$$

Let us now look for the fixed points of Eq. (5). Introduce first the notation $\alpha=\Omega-\Delta E$. From the system (5) we find the following equation for the fixed points:

$$
\begin{aligned}
& z_{c}=\left(1-\frac{\eta^{2} \Omega^{2}}{K_{1}^{2} \cos \Delta_{c}^{2}}\right)^{1 / 2}, \\
& \alpha=z_{c}\left(\lambda-K_{1}^{2} \frac{\sin 2 \Delta_{c}}{2 \eta \Omega}\right) .
\end{aligned}
$$

For small $z_{c}, z_{c}^{2} \ll 1$, we can find the explicit solution

$$
\begin{gathered}
\Delta_{c}=\arccos \left(\frac{\eta \Omega}{K_{1}}\right), \\
z_{c} \approx \frac{\Omega-\Delta E}{\Lambda-\sqrt{K_{1}^{2}-\eta^{2} \Omega^{2}}} .
\end{gathered}
$$

The existence of a stationary solution is connected with the circumstance that the damping of the oscillation in quantum tunneling is compensated by the periodic variation of the trap. In Ref. [6] the influence of the damping on the macroscopic quantum interference has been investigated numerically and degradation of the interference due to damping has been analyzed. The predicted stationary solutions, where these effects are compensated by the variable driving, are indeed phase-locked solutions. Thus we can think that the fluctuations of the relative phase will be suppressed in such states. It is an important prediction since BEC's have phase fluctuations and it is difficult to maintain the constant relative phase of condensates. This phenomenon can be useful to develop the phase standard for the Bose-Einstein condensate- a problem attracting considerable attention recently [19].

We can estimate the value of fixed points for an experimental situation. For example, when $\Omega=0.7, \eta=0.1, K_{1}$ $=0.2$, and $\Lambda=2.5$, we obtain the fixed points $z_{1 c}=0.2599$ and $z_{2 c}=0.3037$. Stability analysis shows that only the first point is stable and corresponds to the global attractor. The results of the numerical simulations of the initial system for the relative population $z(t)$ are presented in Fig. 1. It shows that the agreement between theory and numerical simulations is good. 


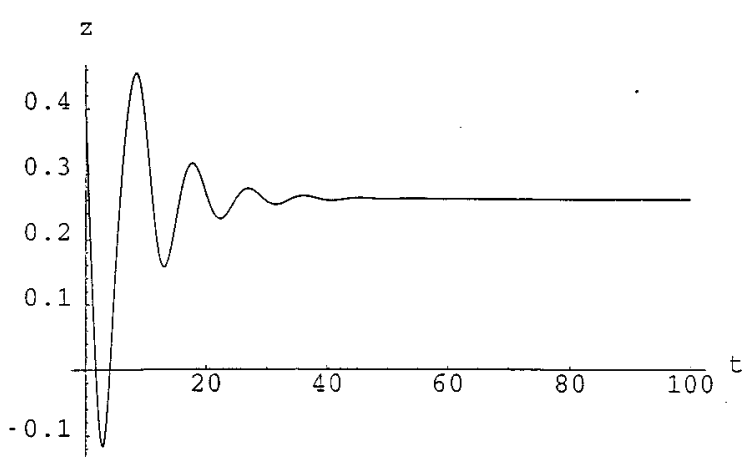

FIG. 1. The typical behavior of the solutions to Eq. (2) when the damping is taken in the form $\Gamma=\eta \phi_{t}$. This case corresponds to $\Omega=0.7, \eta=0.1, K_{1}=0.2$, and $\Lambda=2.5$. The theoretical value is $z_{c}$ $=0.2599$, the numerical value is $z_{c}=0.26$.

The same system of equations also describes the nonlinear Josephson-type oscillations in the relative population of a driven, two-component Bose-Einstein condensate. The twocomponent condensates are those in which two different hyperfine states can be populated and confined in the same trap. A weak driving field couples two internal states and as a result the oscillations in the relative population will occur [20]. In this case the damping term has the form $\Gamma=-\eta z$. Repeating the above-mentioned procedure, we obtain the averaged system

$$
\begin{gathered}
\bar{z}_{t}=-K_{1} \sqrt{1-\bar{z}^{2}} \cos \bar{\Delta}-\eta \bar{z}, \\
\bar{\Delta}_{t}=\Lambda \bar{z}+\Delta E-\Omega-\frac{K_{1} \bar{z}}{\sqrt{1-\bar{z}^{2}}} \sin \bar{\Delta} .
\end{gathered}
$$

In the model [20], $\Delta E$ corresponds to $\mu_{2}-\mu_{1}-\kappa$, where $\mu_{i}$ are chemical potentials and $\kappa$ is frequency detuning $\kappa$ $=\omega_{d}-\omega_{0}, \omega_{0}$ is the separation in frequency of two hyperfine states, and $\omega_{0}$ is the frequency of drive. The fixed points are

$$
\begin{gathered}
\bar{\Delta}_{c}=\arccos \left(\frac{\eta z_{c}}{K_{1} \sqrt{1-z_{c}^{2}}}\right), \\
\Lambda z_{c}-\alpha-\frac{K_{1} z_{c}}{\sqrt{1-z_{c}^{2}}}\left(1-\frac{\eta^{2} z_{c}^{2}}{K_{1}^{2}\left(1-z_{c}^{2}\right)}\right)^{1 / 2} .
\end{gathered}
$$

For the case of small $z_{c}^{2} \ll 1$ we have the estimate for $z_{c}$,

$$
z_{c}=\frac{\Delta E-\Omega}{\Lambda-K_{1}}+\frac{(\Delta E-\Omega)^{3}\left(K_{1}^{2}-\eta^{2}\right)}{2 K_{1}\left(\Lambda-K_{1}\right)^{4}} .
$$

The typical values of the parameters are $\Omega=0.7, \mu_{2}$ $-\mu_{1}-\kappa=\Delta E=1, \eta=0.1, K_{1}=0.2$, and $\Lambda=4$, and the fixed points are $z_{3 c}=-0.111$ and $z_{4 c}=-0.13$. The analysis shows that the first fixed point is stable and second is unstable.

In Fig. 2 we present the plot of solution of the full system

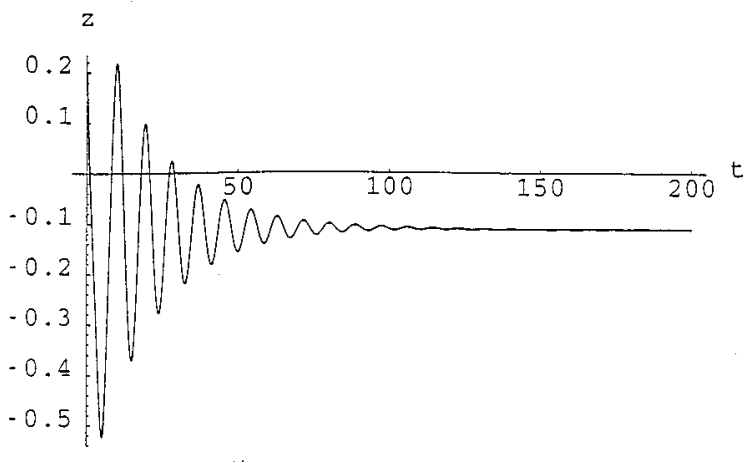

FIG. 2. The typical behavior of the solutions to Eq. (2) when the damping is taken in the form $\Gamma=\eta z$. This case corresponds to $\Omega$ $=0.7, \Delta E=1, \eta=0.1, K_{1}=0.2$, and $\Lambda=2.5$.

for the damping term $\eta z$ with the above-mentioned set of parameters. All solutions decay to the first fixed point.

\section{CHAOTIC OSCILLATIONS IN RELATIVE ATOMIC POPULATION}

In this section we consider the interference in the system which in some limit is mathematically equivalent to the motion of a particle in a double-well potential under parametric periodic perturbations. The particle motion is of course unharmonic and the nonlinear resonances between oscillations of a particle and oscillations of the trap potential are possible. One of consequences of this is the possible appearance of chaotic dynamics in such systems. We will now study the conditions for the appearance of the chaotic dynamics in atomic tunneling.

To proceed, it will be useful first to consider some aspects of the unperturbed system, that is, when the coupling is constant. In this case the system (2) is equivalent to the quartic (Duffing) oscillator [6,23].

We can write the equation for $z(t)$ as

$$
z_{t t}=-\frac{\partial V}{\partial z}
$$

where the potential $V(z)$ is given by

$$
V(z)=z^{2}\left(a+b z^{2}\right), \quad a=2 K_{0}^{2}-\frac{\Lambda H}{2}, \quad b=\frac{\Lambda^{2}}{8},
$$

and $H$ is the Hamiltonian. The total energy of the effective particle is

$$
E_{0}=\frac{z_{t}^{2}}{2}+V(z)=2 K^{2}-H^{2} / 2
$$

When $E_{0}>0$, we have the oscillating regime with $\langle z(t)\rangle$ $=0$ (nonlinear Rabi oscillations). This case corresponds to the periodic flux of atoms from one BEC to the other. When $E_{0}<0$, the motion of the effective particle is confined in that of the wells. This case corresponds to the localization of atomic population in one of the condensates-the so-called macroscopic quantum self-trapping (MQST). The case $E_{0}$ 
$=0$, i.e., $H=2 K$, corresponds to the separatrix solution separating these two regimes. It is interesting to investigate the dynamics of atomic population, when the inital parameters $z(0), \Phi(0)$ are close to the separatrix of the unperturbed system. From the general theory of nonlinear driven oscillations [21], we can expect the appearance of chaotic macroscopic tunneling phenomena.

The separatrix solutions for the right-hand side well (condensate) are

$$
\begin{gathered}
z_{s}(t)=\sqrt{a / b} \operatorname{sech}(\sqrt{2 a} t), \\
\sin ^{2}\left(\Phi_{s}\right)=\frac{\sigma^{2} \operatorname{sech}^{2}(\sqrt{2 a} t) \tanh ^{2}(\sqrt{2 a} t)}{2 K_{0}^{2}\left[b-a \operatorname{sech}^{2}(\sqrt{2 a} t)\right]},
\end{gathered}
$$

where $H=H_{s}=2 \mathrm{~K}$.

The motion near the separatrix is very sensitive to the change of the initial condition and a stochastic layer appears, leading to the chaotic dynamics of the solutions of the system (2). The Melnikov function method is effective to find the regions of chaotic behavior [21,22]. The system (2) may written in the form $\mathbf{r}_{t}=\mathbf{f}+\epsilon \mathbf{g}$ and has a hyperbolic fixed point. According to this method, we need to calculate the Melnikov function $M\left(t_{0}\right)$,

$$
M\left(t_{0}\right)=\int_{-\infty}^{\infty}\left[f_{2 s} g_{1 s}\left(t, t_{0}\right)-f_{1 s} g_{2 s}\left(t, t_{0}\right)\right] d t
$$

As is well known, the existence of zeros of $M\left(t_{0}\right)$ indicates the intersection of separatrices and the existence of homoclinical chaos. Substituting the expressions for $f_{i}, g_{i}$ from the system (2) and calculating the integrals, we find the final expressions for the Melnikov function. We shall give the results for two possible damping terms.

\section{A. The case of the damping in the form $\boldsymbol{\eta} \Phi_{t}$}

The integrations in Eq. (16) are cumbersome, and will be omitted. The final result for the Melnikov function is

$$
M_{1}\left(t_{0}\right)=-K_{1} F_{1} \cos \left(\Omega t_{0}\right)-F_{2} \eta,
$$

where

$$
\begin{gathered}
F_{1}=\frac{\pi \Lambda \Omega^{2}}{4 K_{0} b \sinh \left(\frac{\pi \Omega}{2 \sqrt{2 a}}\right)}, \\
F_{2}=\frac{2 \Lambda \sqrt{2 a}}{\sqrt{a b-a^{2}}}\left(\Lambda-2 H_{s}\right) \arctan [\sqrt{a /(b-a)}] \\
-\frac{\sqrt{2 a}}{16(b-a) b^{2}} G_{1},
\end{gathered}
$$

where

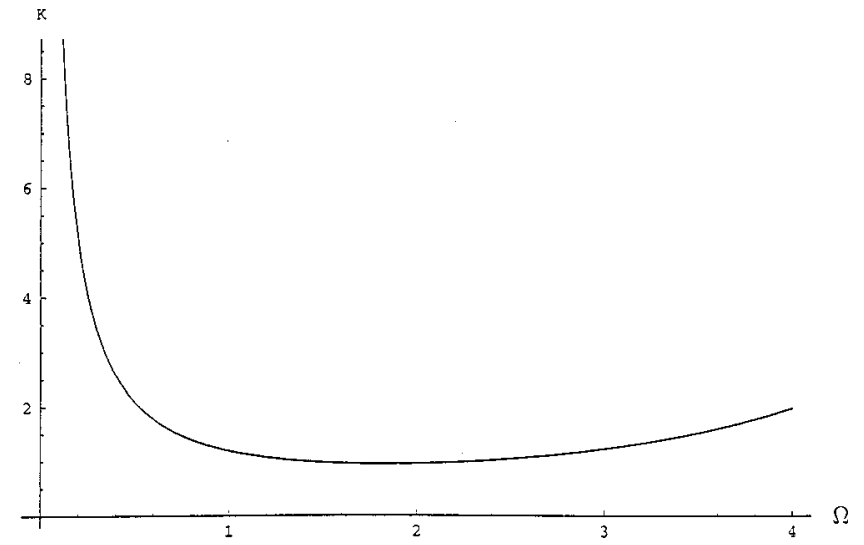

FIG. 3. The regions of regular and chaotic oscillations in plane $K_{1}, \Omega$ from the Melnikov approach.

$$
\begin{aligned}
G_{1}= & {\left[-2 b^{2}\left(2 H_{s}-\Lambda\right)\left(b H_{s}-4 a \Lambda+3 b \Lambda\right)\right] } \\
& \times \frac{\arctan \left(a / \sqrt{a b-a^{2}}\right)}{\sqrt{a b-a^{2}}} \\
& -2\left[4 a^{2} \Lambda^{2}-5 a b \Lambda^{2}+2 b^{2}\left(4 H_{s}^{2}-4 H_{s} \Lambda+3 \Lambda^{2}\right)\right] .
\end{aligned}
$$

The simple zero of $M\left(t_{0}\right)$ is absent if the condition

$$
\eta>\frac{F_{1}}{F_{2}}
$$

is satisfied. In Fig. 3 we plot this criterion in the $\left(K_{1}, \Omega\right)$ plane for $\eta=0.1$. Regions below the curve correspond to regular oscillations and those above correspond to chaotic oscillations of $z(t)$. The criterion gives a good lower bound on the region of chaos in this plane. We verified this lower bound in the $\left(K_{1}, \Omega\right)$ plane by numerical simulations and found a good agreement with the formulas (20).

When the damping coefficient is zero, we have the expression for the width of stochastic layer near the separatrix as

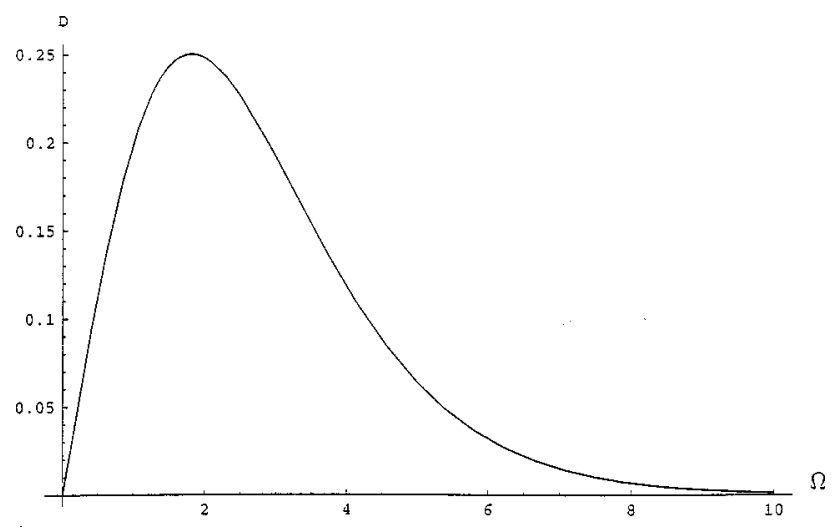

FIG. 4. The width of stochastic layer as a function of frequency $\Omega$, when $\eta=0, K_{0}=1, K_{1}=0.2, \Lambda=9.9, z(0)=0.6$, and $\Phi(0)$ $=0$. 




FIG. 5. The typical oscillations of the relative population $z(t)$ for $K_{1}=1.2, \Lambda=9.9, z(0)=0.6, \Phi_{0}=0$, and $\Omega=1.5$.

$$
D=\frac{\pi \Lambda K_{1} \Omega^{2}}{4 K_{0} b \sinh \left(\frac{\pi \Omega}{2 \sqrt{2 a}}\right)}
$$

In Fig. 4 we plot the width of the stochastic layer as a function of the frequency for $K_{1}=0.2, \Lambda=9.9, z(0)=0.6$, and $\Phi(0)=0$. The maximum of the width is given by the solution of the equation

$$
\Omega \tanh \left(\frac{\pi \Omega}{2 \sqrt{2 \alpha}}\right)=\frac{4 \sqrt{2 a}}{\pi} .
$$

For this choice of parameters, we have $\Omega_{m}=1.89$. For $\Omega$ $\geqslant 2, a \geqslant 1$ we get the estimate $\Omega_{m} \approx 4 \sqrt{2 a} / \pi$. It can be seen that, for the high frequencies $\Omega \gg \sqrt{a}$, the stochastic layer is exponentially narrow and motion is regular. The analysis of this case will be performed in detail in the next section. In Fig. 5 we plot the typical oscillations in the relative atomic population for $\Lambda=9.9, K_{0}=1, K_{1}=0.2, z(0)=0.6$, and $\Phi(0)=0$ for $\Omega=1.5$. It turns out that for $\Omega$ between 1.5 and 3.22 we have chaotic oscillations, and for small or large $\Omega$, regular oscillations. In Fig. 6 the phase portrait is given. In Fig. 7 the influence of the damping on chaos is illustrated. Parameters are the same as in Fig. 5 and $\eta=0.15$. In this case the oscillations become regular.

$\Phi$

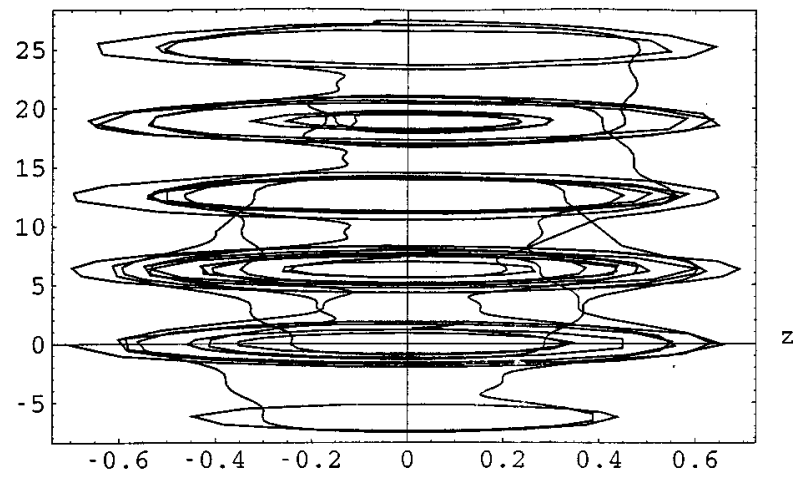

FIG. 6. The phase portrait in the $(\Phi, z)$ plane for the same values of the parameters as in Fig. 5.



FIG. 7. The typical oscillations of the relative population $z(t)$ for $K_{1}=0.2, \Lambda=2.5, z(0)=0.9, \Phi_{0}=\pi$, and $\Omega=3.22$.

\section{B. The case of the damping term in the form $\eta z(t)$}

The calculation of the integrals which give the Melnikov function is again cumbersome, and one finds the following result:

$$
M\left(t_{0}\right)=F_{1} \cos \left(\Omega t_{0}\right)+\eta F_{3},
$$

where

$$
F_{3}=\frac{\Lambda-2 H_{s}}{\sqrt{2(b-a)}} \arctan [\sqrt{a /(b-a)}]+\frac{3 \Lambda \sqrt{a}}{2 \sqrt{2} b} .
$$

The zeros of $M\left(t_{0}\right)$ are absent when the condition

$$
\eta>\frac{F_{1}}{F_{3}}
$$

is satisfied.

If we consider for example the case when $K_{1}=0.2, \Omega$ $=0.7, \Lambda=2.5, z(0)=0.6$, and $\Phi(0)=\pi$, the damping constant should be $\eta=0.15$. In Figs. 7 and 8 the chaotic and periodic oscillations of the relative population in the dependence of the frequency for fixed $z(0), \Phi(0)$ are plotted.

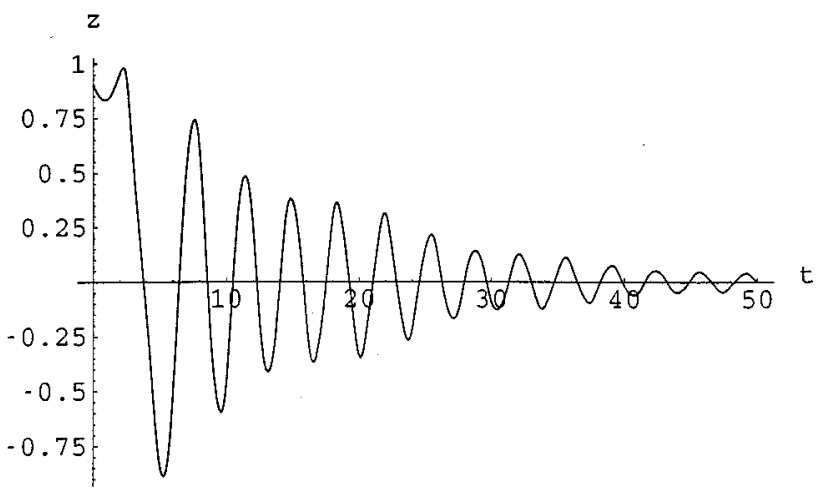

FIG. 8. The typical oscillations of $z(t)$ for the same set of parameters as in Fig. 7, but with $\Gamma=-0.15 z$. 


\section{RAPIDLY VARYING TRAP POTENTIAL}

In the rapidly varying case $K=K(t / \epsilon)$ we can apply the averaging techniques based on the multiscale expansions [24-26]. Let us search the solution in the form of the sum of the slowly varying components $U, V$ and rapidly varying corrections $u_{i}, v_{i}$,

$$
\begin{aligned}
& \psi_{1}(t)=U\left(t_{k}\right)+\epsilon u_{1}(\zeta)+\epsilon^{2} u_{2}(\zeta)+\cdots, \\
& \psi_{2}(t)=V\left(t_{k}\right)+\epsilon v_{2}(\zeta)+\epsilon^{2} v_{2}(\zeta)+\cdots
\end{aligned}
$$

where $\zeta=t / \epsilon, \epsilon \ll 1$, and $t_{k}=\epsilon^{k} t$ are slow times. The averages over a period of rapid oscillations are $\left\langle u_{i}\right\rangle=\left\langle u_{i}\right\rangle=0$. The functions $u_{i}, v_{i}$ are assumed to be functions of the rapid time $\zeta$ and the slow varying functions $U, V$. The derivatives can be written in the form

$$
\frac{d U}{d t}=\frac{d U}{d t_{0}}+\epsilon \frac{d U}{d t_{1}}+\cdots
$$

and analogously for the derivative of $V$.

Below, we will assume $E_{1}=E_{2}$. Then the time dependence in $E(t)$ can be removed by a simple field transformation $u(v)=u(v) \exp \left((i / h) \int E\left(t^{\prime}\right) d t^{\prime}\right)$. In the zeroth order in $\epsilon$ we get the equations

$$
\begin{aligned}
& i \hbar\left(\frac{\partial u_{0}}{\partial t_{0}}+\frac{\partial u_{1}}{\partial \zeta}\right)=E u_{0}-K(\zeta) v_{0}+\alpha\left|u_{0}\right|^{2} u_{0}, \\
& i \hbar\left(\frac{\partial v_{0}}{\partial t_{0}}+\frac{\partial u_{1}}{\partial \zeta}\right)=E v_{0}-K(\zeta) u_{0}+\alpha\left|v_{0}\right|^{2} v_{0}
\end{aligned}
$$

Requiring the exclusion of the secular in $\zeta$ terms, we find the corrections $u_{1}, v_{1}$,

$$
\begin{gathered}
u_{1}=\frac{i}{\hbar} v_{0}\left(\mu_{1}-\left\langle\mu_{1}\right\rangle\right), \\
v_{1}=\frac{i}{\hbar} u_{0}\left(\mu_{1}-\left\langle\mu_{1}\right\rangle\right),
\end{gathered}
$$

where $\mu_{1}=\int_{0}^{\zeta}[K(s)-\langle K\rangle] d s$ and the system for $u_{0}, v_{0}$ becomes

$$
\begin{aligned}
& i \hbar \frac{\partial u_{0}}{\partial t_{0}}=E u_{0}-\langle K\rangle v_{0}+\alpha\left|u_{0}\right|^{2} u_{0}, \\
& i \hbar \frac{\partial v_{0}}{\partial t_{0}}=E v_{0}-\langle K\rangle u_{0}+\alpha\left|v_{0}\right|^{2} v_{0} .
\end{aligned}
$$

In the order $\epsilon$ we have

$$
\frac{\partial u_{0}}{\partial t_{1}}=0, \quad \frac{\partial v_{0}}{\partial t_{1}}=0
$$

and we obtain, for the second-order corrections $u_{2}, v_{2}$; the elimination of the secular terms result in

$$
\begin{aligned}
u_{2}= & -\frac{i}{\hbar}\left(\mu_{2}-\left\langle\mu_{2}\right\rangle\right)\left(\frac{\partial v_{0}}{\partial t_{0}}+\frac{i}{\hbar} v_{0}+\frac{i \alpha}{\hbar}\left[2\left|v_{0}\right|^{2} v_{0}+u_{0}^{2} v_{0}^{*}\right]\right) \\
& -\left[\left(\mu_{1}-\left\langle\mu_{1}\right\rangle\right)^{2}-2 M\right] u_{0}, \\
v_{2}= & -\frac{i}{\hbar}\left(\mu_{2}-\left\langle\mu_{2}\right\rangle\right)\left(\frac{\partial u_{0}}{\partial t_{0}}+\frac{i}{\hbar} u_{0}+\frac{i \alpha}{\hbar}\left[2\left|v_{0}\right|^{2} u_{0}+v_{0}^{2} u_{0}^{*}\right]\right) \\
& -\left[\left(\mu_{1}-\left\langle\mu_{1}\right\rangle\right)^{2}-2 M\right] v_{0},
\end{aligned}
$$

where

$$
\mu_{2}=\int_{0}^{\zeta}\left(\mu_{1}(s)-\left\langle\mu_{1}\right\rangle\right) d s, \quad M=\left(\left\langle\mu_{1}^{2}\right\rangle-\left\langle\mu_{1}\right\rangle^{2}\right) .
$$

From this order we get the equations

$$
\begin{aligned}
& \frac{\partial u_{0}}{\partial t_{2}}=-\frac{2 i M \alpha}{h^{3}}\left[2\left|v_{0}\right|^{2} u_{0}-v_{0}^{2} u_{0}^{*}\right], \\
& \frac{\partial v_{0}}{\partial t_{2}}=-\frac{2 i \alpha M}{h^{3}}\left[2\left|u_{0}\right|^{2} v_{0}-u_{0}^{2} v_{0}^{*}\right] .
\end{aligned}
$$

Finally, we have in the next order, $O\left(\epsilon^{2}\right)$, the averaged system

$$
\begin{aligned}
& i \hbar \frac{\partial u_{0}}{\partial t}-E u_{0}-\alpha\left|u_{0}\right|^{2} u_{0} \\
& \quad=-\langle K\rangle u_{0}+2 \frac{\epsilon^{2} \alpha M}{\hbar^{2}}\left[2\left|v_{0}\right|^{2} u_{0}-v_{0}^{2} u_{0}^{*}\right], \\
& i \hbar \frac{\partial u_{0}}{\partial t}-E v_{0}-\alpha\left|v_{0}\right|^{2} v_{0} \\
& \quad=-\langle K\rangle v_{0}+2 \frac{\epsilon^{2} \alpha M}{\hbar^{2}}\left[2\left|u_{0}\right|^{2} v_{0}-u_{0}^{2} v_{0}^{*}\right] .
\end{aligned}
$$

For periodic modulations of tunnel coupling, we obtain $M$ $=K_{1}^{2} / \Omega^{2}$.

This averaged system is the main result of this section. We see that, in comparison with the constant coupling case, new effects appear. First, the cross term appears, corresponding to a change of type in the effective nonlinearity in coupled BEC dynamics. Second, there appears an additional nonlinear phase-sensitive coupling. This term leads to new minima in the effective potential picture. In the constant coupling case, the oscillations of $z(t)$ are described by the Duffing oscillator. Now we have a more complicated nonlinear oscillator, describing more complicated interference patterns.

\section{ANALYSIS OF THE AVERAGED DYNAMICS}

Using the variables introduced in Sec. II, we get the system

$$
z_{t}=-2\langle K\rangle \sqrt{1-z^{2}} \sin (\Phi)-\nu \delta\left(1-z^{2}\right) \sin (2 \Phi),
$$




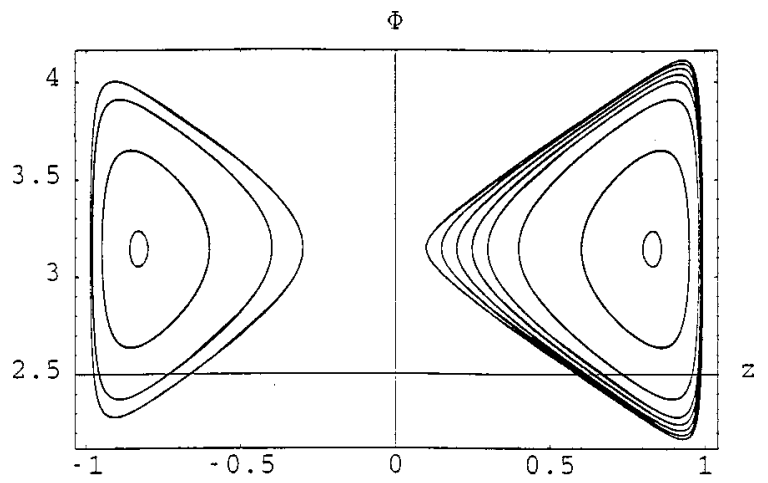

FIG. 9. The phase portrait in a plane $z, \Phi$ of the averaged system of equations (40) for $\Phi(0)=\pi, \Lambda=2.0$, and $\delta=0.2$.

$$
\Phi_{t}=\Lambda z+2 \frac{\langle K\rangle z}{\sqrt{1-z^{2}}} \cos (\Phi)-\nu \delta z[2-\cos (2 \Phi)],
$$

where we have defined $\delta=\epsilon^{2} \alpha M / h^{2}$. This system has the following Hamiltonian:

$$
H=\frac{\Lambda_{1} z^{2}}{2}-2\langle K\rangle \sqrt{1-z^{2}} \cos (\Phi)-\frac{\delta}{2}\left(1-z^{2}\right) \cos (2 \Phi),
$$

where $\Lambda_{1}=\Lambda-2 \delta$.

When the parameter $\delta=0$, we get back the system (2). The effective particle motion is that of the double nonrigid pendulum system.

Let us consider the different regimes of oscillations.

\section{A. Linear regime}

This is the case where $|z| \ll 1,|\Phi| \ll 1$. Then, from the system (40), it follows that

$$
z_{t t}=-2(\langle K\rangle+\delta)(\Lambda+2\langle K\rangle-\delta) z .
$$

Then the frequency of linear oscillations of the number of atoms is

$$
\omega_{L}^{2}=-2(\langle K\rangle+\delta)(\Lambda+2\langle K\rangle-\delta) .
$$

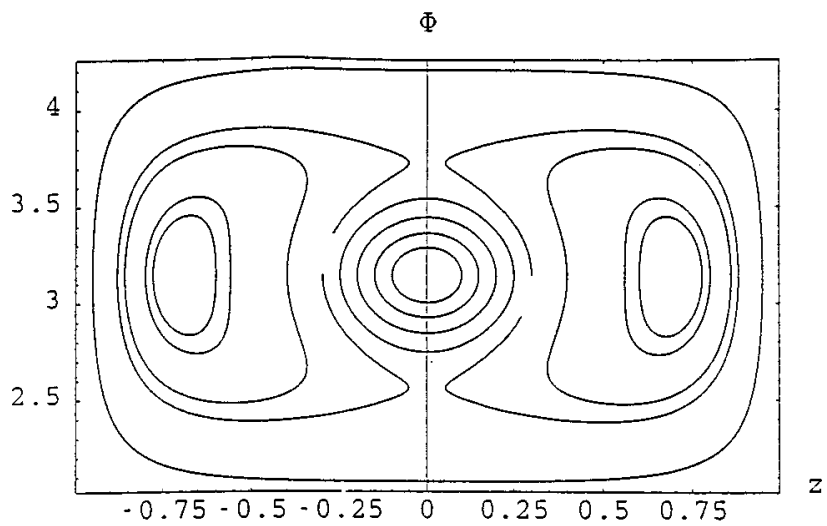

FIG. 10. The phase portrait in a plane $z, \Phi$ of the averaged system of equations (40) for $\Phi(0)=\pi, \Lambda=2.0$, and $\delta=0.6$.

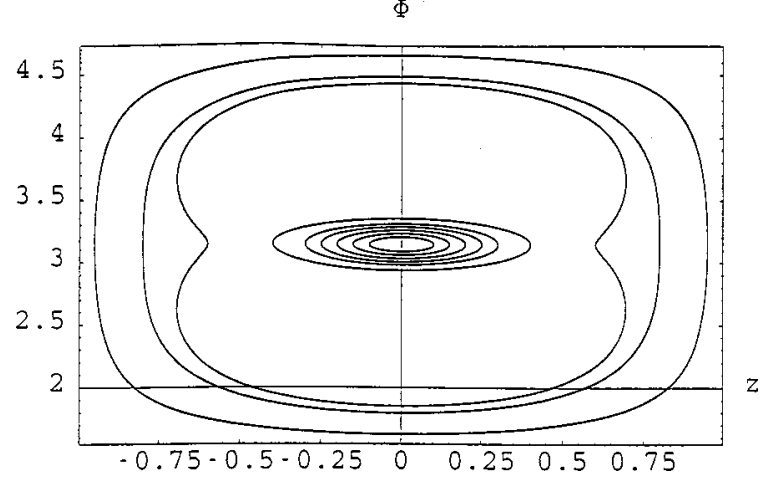

FIG. 11. The phase portrait in a plane $z, \Phi$ of the averaged system of equations (40) for $\Phi(0)=\pi, \Lambda=2.0$, and $\delta=0.8$.

Thus, the frequency of the linear oscillations grows with $\delta \omega^{2}=\omega_{L}^{2}-\omega_{0}^{2}=2 \delta(\langle K\rangle+\Lambda-\delta)$, in comparison with a nonmodulated case.

\section{B. $z^{2} \ll 1$, and $\Phi$ is not small}

The phase dynamics is described by the equation

$$
\begin{aligned}
\Phi_{t t}+ & 2\langle K\rangle(\Lambda-2 \delta) \sin (\Phi)+\left(\delta \Lambda+2\langle K\rangle^{2}-2 \delta^{2}\right) \sin (2 \Phi) \\
& +2 \delta\langle K\rangle \sin (3 \Phi)+\frac{\delta^{2}}{2} \sin (4 \Phi)=0 .
\end{aligned}
$$

At derivation of this equation we take into account that $\Phi_{t}$ $\sim z$, so we neglect by terms $\sim z \Phi_{t}$. This equation is equivalent to a generalized pendulum motion whose effective potential is given

$$
\begin{aligned}
U(\Phi)= & -2\langle K\rangle(\Lambda-2 \delta)-\frac{1}{2}\left(\Lambda \delta+2\langle K\rangle^{2}-2 \delta^{2}\right) \cos (2 \Phi) \\
& -\frac{2 \delta\langle K\rangle}{3} \cos (3 \Phi)-\frac{\delta^{2}}{8} \cos (4 \Phi)
\end{aligned}
$$

The additional minima at $\Phi= \pm n \pi$ correspond to the trapping of oscillations in states with locked phase.

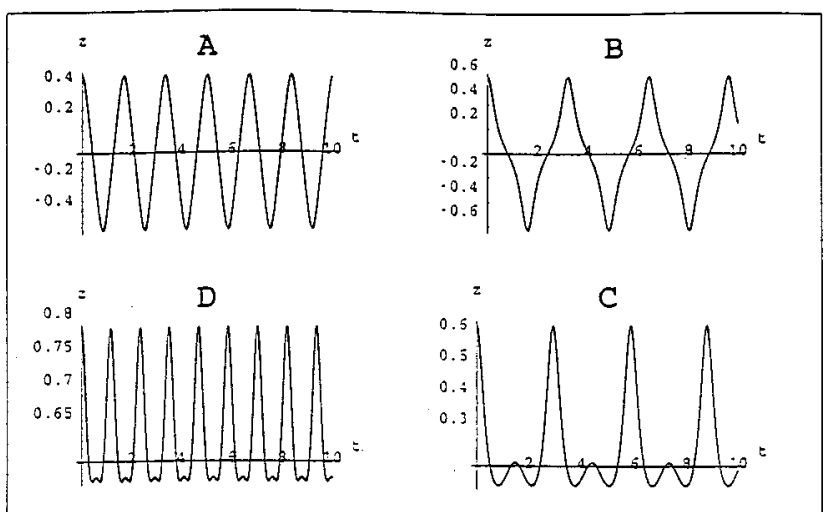

FIG. 12. The typical behavior of the solutions to Eq. (2), for $\delta$ $=0.8$ and $\Lambda=10, \Phi(0)=0$ and varying $z(0)$. (A) corresponds to $z(0)=0.5,(B)$ to $z(0)=0.65,(C)$ to $z(0)=0.68$, and $(D)$ to $z(0)$ $=0.80$. $(B)$ is just before the localization transition and $(C)$ just after. 


\section{MACROSCOPIC QUANTUM SELF-TRAPPING}

This phenomenon consists in the self-trapping of the atomic number in one of the condensates, depending on the initial difference in atomic number and on the relative phase of condensates. This is connected with the phase-sensitive linear coupling. The nonlinear guided wave optics analog of this phenomenon, first predicted in [4], is the switching of the power in the dual core couplers from one core to the other, depending on the initial power or the phase difference. The additional coupling in the averaged system (40) is nonlinear and also phase-sensitive. Thus the condition of localization of atomic population should be modified in comparison with the one considered earlier.

Let us find the fixed points of the averaged system (40). The first group is for $\Phi_{n}=\pi n, n=0, \pm 1, \pm 2, \ldots$ For each $\Phi_{n}$ there can exist one or three roots for $z$. One of the roots is $z_{1}=0$, two others are

$$
z_{s 1,2}= \pm\left(1-\frac{4\langle K\rangle^{2}}{(\Lambda-\delta)^{2}}\right)^{1 / 2} .
$$

Note that if $(\Lambda-\delta)\langle K\rangle<0$, then three roots exists for even $n$ and one root $\left(z_{1}=0\right)$ exists for odd $n$. For $(\Lambda-\delta)\langle K\rangle>0$, one has the opposite situation. The critical value is $\Lambda_{c}$ $>2\langle K\rangle+\delta$.

One can show, however, that linear stability analysis predicts that this set is unstable when $\delta>\Lambda / 2$.
The second group of fixed points is given by

$$
\cos (\Phi)=-\frac{\langle K\rangle}{\sqrt{1-z^{2}}} .
$$

Again, there are two possibilities.

(i) $\Lambda=3 \delta$, where the whole line is stationary.

(ii) $\Lambda \neq 3 \delta$, where one has the following static point:

$$
\cos (\Phi)=-\frac{\langle K\rangle}{\delta}, \quad z=0
$$

This root assumes that $|\langle K\rangle / \delta|<1$. The linear stability analysis shows that the fixed points are unstable when $\Lambda>3 \delta$.

Following [6], from the constraint coming from the energy conservation and the boundness of the tunneling energy, given by

$$
\begin{aligned}
H= & \frac{\Lambda_{1} z^{2}(0)}{2}-2\langle\langle K\rangle\rangle \sqrt{1-z^{2}(0)} \cos (\Phi(0)) \\
& -\frac{\delta}{2}\left[1-z^{2}(0)\right] \cos (2 \Phi(0)) \geqslant 2\langle K\rangle-\frac{\delta}{2},
\end{aligned}
$$

we obtain the estimate for the critical value of $\Lambda_{c}$ when the self-localization occurs. We find that

$$
\Lambda_{c}=\frac{4\langle K\rangle\left[\sqrt{1-z^{2}(0)} \cos \Phi(0)+1\right]+\delta\left(\left[1-z^{2}(0)\right] \cos 2 \Phi(0)-1\right)}{z^{2}(0)}+2 \delta .
$$

The phase portrait is plotted in Figs. 9-11 for initial phase difference $\Phi(0)=\pi$ and $\Lambda=2.5$, for different $\delta$ $=0.2,0.6,0.8$. The regions corresponding to different dynamics of $z(t)$ with $\langle z\rangle=0$ and $\langle z\rangle \neq 0$ are shown. The increasing of $\delta$ (i.e., $K_{1} / \Omega$ ) leads to the distortion of the MQST regime, and for $\delta \sim 1$ to the nonlinear Rabi-like oscillations. The typical oscillations in time of the relative population for different values of $\delta$ and for fixed $\Lambda$ are plotted in Fig. 12. The influence of rapid modulations leads to the appearance of new minima in the oscillations. This can be described as a result of the overlap of two double-well potentials. This conclusion is also confirmed by the results of numerical simulations of the GP equation for the single BEC under the rapidly varying trap potential performed in [18]. In this work, it has been shown that the effect of rapid modulations is the appearance of an effective double-well trap potential.

\section{CONCLUSION}

In this paper we have studied the new effects coming the time variation of the trap potential and from damping on the nonlinear oscillations in the relative population behavior between two BEC's. For the slowly varying trap we predict synchronization of oscillations of the trap with oscillations of the relative population. We find the fixed points for both types of damping terms occurring in the studies of two coupled condensates. Using the Melnikov approach, we study the possibility of the appearance of the chaotic oscillations in the tunneling phenomena between two coupled Bose-Einstein condensates. We calculate the width of the stochastic layer in phase space, in which the dynamics is chaotic. We find the lower bound on the region of chaos in the $\left(K_{1}, \Omega\right)$ plane. We obtain the estimate for the damping coefficient when the chaos is suppressed. For the rapidly varying trap we use the multiscale method and derive the averaged equations for coupled modes describing the tunneling phenomena. We find the fixed points in this case describing the stationary states in two coupled BEC's. The expression for the critical value of the nonlinearity parameter, when the macroscopic quantum self-trapping occurs, is derived [26]. Our results show that there is interest to study the contribution of the quantum tunneling for this driven case and the investigation of resonances and chaos in oscillations of atomic population for the strongly overlapped condensates [27]. These problems require separate investigation.

\section{ACKNOWLEDGMENTS}

The authors are grateful to E. N. Tsoy for useful discussions and comments. This work was partially supported by an FAPESP Grant. 
[1] M. R. Andrews, C. G. Townsend, and W. Ketterle, Science 275, 637 (1997).

[2] J. Javanainen, Phys. Rev. Lett. 57, 3164 (1986).

[3] F. Dalfovo, L. Pitaevski, and S. Stringari, Phys. Rev. A 54, 4213 (1996).

[4] A. Smerzi, S. Fantoni, S. Giovanazzi, and S. R. Shenoy, Phys. Rev. Lett. 79, 4950 (1997).

[5] G. J. Milburn, J. Corney, E. M. Wright, and D. F. Walls, Phys. Rev. A 55, 4318 (1997).

[6] S. Raghavan, A. Smerzi, S. Fantoni, and S. R. Shenoy, Phys. Rev. A 59, 620 (1999).

[7] I. Zapata, F. Sols, and A. Leggett, Phys. Rev. A 57, R28 (1998).

[8] I. Mazino, S. Raghavan, S. Fantoni, S. R. Shenoy, and A. Smerzi, Phys. Rev. A 60, 487 (1999).

[9] A. Barone and B. Paterno, Physics and Applications of the Josephson Effect (Wiley, New York, 1982).

[10] S. M. Jensen, IEEE J. Quantum Electron. 18, 1580 (1982); A. A. Maier, Sov. J. Quantum Electron. 12, 1490 (1982).

[11] F. Kh. Abdullaev, S. A. Darmanyan, and P. K. Khabibullaev, Optical Solitons (Springer, Heidelberg, 1993).

[12] S. Trillo, S. Wabnitz, E. M. Wright, and G. Stegeman, Opt. Lett. 13, 871 (1988).

[13] F. Kh. Abdullaev, R. M. Abrarov, and S. A. Darmanyan, Opt. Lett. 14, 131 (1989).

[14] S. A. Darmanyan, Opt. Commun. 90, 301 (1992).
[15] I. M. Skinner, G. D. Peng, and B. A. Malomed, Opt. Commun. 113, 493 (1995).

[16] Y. Castin and R. Dum, Phys. Rev. Lett. 77, 5315 (1996).

[17] J. J. Garsia-Ripoll, V. M. Perez-Garsia, and P. Torres, Phys. Rev. Lett. 89, 1715 (1999).

[18] R. Dum, A. Sampera, K.-A. Suominen, M. Brewczyk, M. Kus, K. Rzazewski, and M. Lewenstein, Phys. Rev. Lett. 80, 3899 (1998).

[19] J. A. Dunningham and K. Burnett, Phys. Rev. Lett. 82, 3729 (1999).

[20] J. Williams, R. Walser, J. Cooper, E. Cornell, and M. Holland, Phys. Rev. A 59, R31 (1999).

[21] J. Guckenheimer and P. Holmes, Nonlinear Oscillations, Dynamical Systems, and Bifurcations of Vector Fields (SpringerVerlag, New York, 1983).

[22] F. Kh. Abdullaev, Phys. Rep. 179, 1 (1989).

[23] V. M. Kenkre and G. P. Tsironis, Phys. Rev. B 35, 1473 (1987).

[24] J. Kevorkian and J. D. Cole, Perturbation Methods in Applied Mathematics (Springer, New York, 1981).

[25] T. S. Yang and W. L. Kath, Opt. Lett. 22, 985 (1997).

[26] F. Kh. Abdullaev, Theory of Solitons in Inhomogeneous Media (Wiley, Chichester, 1994).

[27] E. Ostrovskaya, Y. S. Kivshar, M. Lisak, B. Hall, F. Cattani, and D. Anderson, Phys. Rev. A 61, 031601 (2000). 